\title{
Mass Scales and Their Relations in Symmetric Quantum Field Theory
}

\author{
S. R. Gobira ${ }^{1}$ and M. C. Nemes ${ }^{2}$ \\ (1)UFT - Universidade Federal do Tocantins - Núcleo de Física CEP-77020-210, Palmas - TO - Brazil and \\ (2)UFMG - Universidade Federal de Minas Gerais - Departamento de Física - ICEx - \\ C.P.702, CEP 30.161-970, Belo Horizonte - MG - Brazil \\ Received on 27 May, 2004. Revised version received on 21 March, 2005
}

\begin{abstract}
We illustrate the importance of mass scales and their relation in the specific case of the linear sigma model within the context of its one loop Ward identities. In the calculation it becomes apparent the delicate and essential connection between divergent and finite parts of amplitudes. The examples show how to use mass scales identities which are absolutely necessary to manipulate graphs involving several masses. Furthermore, in the context of the Implicitly Regularization, finite(physical) and divergent (counterterms) parts of the amplitude can and must be written in terms of a single scale which is the renormalization group scale. This facilitates, e.g., obtaining symmetric counterterms and immediately lead to the proper definition of Renormalization Group Constants.
\end{abstract}

\section{INTRODUCTION}

A field theory predictivity, or the ability to obtain results valid to all orders of perturbation theory, relies on logical conditions: the renormalization program has to be a systematic and unambiguously fixed algorithm that satisfies the fundamental properties of locality and causality: it should correspond to the addition of local counterterms to the Lagrangian density. In general, any renormalization procedure involves two steps [1]: 1) A choice of Regularization followed by a subtraction procedure. 2) A set of renormalization conditions in order to define the parameters of theory at a given scale.

This scale acquires a very crucial role. From the most naive point of view it is an arbitrariness coming from the fact that the separation of a divergent amplitude in a finite plus divergent part is defined up to a constant. In several approaches in the literature it appears in different ways, for example in Dimensional Regularization [2] it appears for dimensional reasons. In Differential Renormalization [3] it appears as an integration constant. In our scheme it appears in the rather subtle way, as we will see.

The purpose of this rather technical work is to perform an analytical evaluation of all amplitudes at one loop level which are necessary for an explicit verification of the Ward identities of the chiral linear sigma model with fermions. In this work we illustrate the importance of mass scales and their relation in the specific case of this model within the context of its one loop Ward identities. Besides, we use the unrenormalized amplitude to illustrate the communication between finite(physical) and divergent (counterterms) parts of the amplitude in order to check the Ward identities. The examples show how to use mass scales identities which are absolutely necessary to manipulate graphs involving several masses. Furthermore, in the context of the Implicitly Regularization [4] [5][6], finite(physical) and divergent (counterterms) parts of the amplitude can and must be written in terms of a single scale which is the renormalization group scale. This facilitates, e.g., obtaining symmetric counterterms and immediately leads to the proper definition of Renormalization Group Constants.

Our technique to handle such amplitudes is to assume only implicitly the presence of a regulator in the integrals and algebraically manipulate the integrand until such a separation is achieved. The divergent parts are left in the form of integrals which enable us to recover the result of any regularization prescription.

The reason we have chosen the chiral model is related to the presence of the $\gamma^{5}$ matrix, and to show it can be handled in 4-dimensions without difficulties. The presence of three different masses in this model help us illustrate the scale change mechanism in the context of Ward identities.

This work is organized as follows: in the section II we present the model and the relevant Ward-Takahaski identities. In section III we verify that chiral symmetry helps "taming" the divergent content of this model. In the section IV we briefly recall the Implicit Regularization Prescription. In the section $\mathrm{V}$ we show the mechanism of using the relations between mass scales. Final comments can be found in section VI.

\section{THE LINEAR SIGMA MODEL}

The linear sigma model has a renormalizable Lagrangian constructed by J. Schwinger, J.C [9]. Polkingorne [11], M. Gell-Mann and M.Levy [10]. In this model the fields are: $N_{o}(p, n)$ the nucleon isodublect (fermions), $\overrightarrow{\pi_{o}}\left(\pi_{1}, \pi_{2}, \pi_{3}\right)$ pion isotriplect (pseudoscalar), $\sigma_{o}$ sigma (isoscalar). The chiral symmetric Lagrangian is

$$
\begin{aligned}
\mathcal{L}_{o}= & i \bar{N}_{o} \not \partial N_{o}+\frac{1}{2}\left[\left(\partial_{\mu} \sigma_{o}\right)^{2}+\left(\partial_{\mu} \vec{\pi}_{o}\right)^{2}\right] \\
& -\frac{\mu_{o}^{2}}{2}\left(\sigma_{o}^{2}+\vec{\pi}_{o}^{2}\right)-\frac{\lambda_{o}}{4}\left(\sigma_{o}^{2}+\vec{\pi}_{o}^{2}\right)^{2} \\
& -G_{o} \bar{N}_{o}\left(\sigma_{o}+i \gamma^{5} \vec{\tau} \cdot \vec{\pi}_{o}\right) N_{o}
\end{aligned}
$$

The chiral breaking term is usually

$$
\mathcal{L}_{q}=c_{o} \sigma_{o}
$$

where $c_{o}$ is a parameter.

The explicit chiral symmetry breaking terms, as is well known, will give rise to a nonvanishing vacuum expectation 
value of the sigma field $\left\langle\sigma_{o}\right\rangle=v_{o}$. We therefore perform a shift in order to have the Lagrangian in terms of fields with zero expectation value as below.

$$
s_{o}=\sigma_{o}-v_{o}
$$

The redefined Lagrangian reads

$$
\mathcal{L}_{T}=\mathcal{L}_{F}+\mathcal{L}_{I}
$$

where

$$
\begin{aligned}
\mathcal{L}_{F}= & \bar{N}_{o}\left(i \not \partial-G_{o} v_{o}\right) N_{o} \\
& +1 / 2\left[\left(\partial_{\mu} s_{o}\right)^{2}-\left(\mu_{o}^{2}+3 \lambda_{o} v_{o}^{2}\right) s_{o}^{2}\right] \\
& +1 / 2\left[\left(\partial_{\mu} \vec{\pi}_{o}\right)^{2}-\left(\mu_{o}^{2}+\lambda_{o} v_{o}^{2}\right) \vec{\pi}_{o}^{2}\right]
\end{aligned}
$$

and

$$
\begin{aligned}
\mathcal{L}_{I}= & -G_{o} \bar{N}_{o}\left(s_{o}+i \gamma^{5} \vec{\tau} \cdot \vec{\pi}_{o}\right) N_{o} \\
& -\lambda_{o} / 4\left(s_{o}^{2}+\vec{\pi}_{o}^{2}\right)^{2} \\
& -\lambda_{o} v_{o}\left(s_{o}^{2}+\vec{\pi}_{o}^{2}\right) s_{o}
\end{aligned}
$$

We can now read off the nucleon, pion and sigma meson masses

$$
\begin{gathered}
M_{N}=G_{o} v_{o} \\
M_{\pi}^{2}=\mu_{o}^{2}+\lambda_{o} v_{o}^{2} \\
M_{\sigma}^{2}=\mu_{o}^{2}+3 \lambda_{o} v_{o}^{2}
\end{gathered}
$$

We also define, for purposes of Ward Identities, seven vertices functions, with mesons having zero momentum and the fermions on their mass shell

$$
V_{N \pi N}, V_{N \sigma N}, V_{\pi^{4}}, V_{\sigma^{4}}, V_{\pi^{2} \sigma^{2}}, V_{\pi^{2} \sigma}, V_{\sigma^{3}}
$$

The indices indicate the interaction involved. The set of Feynman rules we have used are those of Ref. [8] with $F=\langle\sigma\rangle$, $\sigma$ being the renormalized field. The renormalized quantities are introduced as follows,

$$
\begin{aligned}
N_{o} & =\sqrt{Z_{F}} N \\
\left(s_{o}, v_{o}, \vec{\pi}_{o}\right) & =\sqrt{Z_{B}}(s, v, \vec{\pi}) \\
\mu_{o}^{2} & =\frac{1}{Z_{B}}\left(\mu^{2}+\delta \mu^{2}\right) \\
G_{o} & =Z_{g} /\left(Z_{F} \sqrt{Z_{B}}\right) G \\
\lambda_{o} & =\left(Z_{\lambda} / Z_{B}^{2}\right) \lambda
\end{aligned}
$$

which are enough to render the model finite.

\section{A LITTLE HELP FROM CHIRAL SYMMETRY}

Now we will verify that chiral symmetry helps "taming" the divergent content of the model. The Ward Identities relate three point functions (logarithmically divergent) with two points functions (quadratically and linearly divergent). In a general way this fact allows to establish algebraic relations among the subtraction constants of the divergent Green functions of a theory and, therefore, it is a useful tool in the renormalization procedure. Nevertheless Ward Identities can be violated by some used regularization scheme [7] like gauge symmetry in the Quantum Electrodynamics. For the linear sigma model chiral symmetry it is really regularization independent. In an explicit calculation of one loop amplitudes we verify that all quadratically and linearly divergent integrals cancel in the verification of the relative Ward Identities. This cancellation occurs at the level of the evaluation of the Dirac trace and therefore no specific regularization method is required.

We will use the mass parameters $\mu, M$ and $m$ corresponding respectively to the free fields of the pion, sigma and nucleon. The first Ward identity that we consider is

$$
-F\left[V_{\pi^{2} \sigma}(p, 0)\right]=D_{\sigma}^{-1}\left(p^{2}\right)-D_{\pi}^{-1}\left(p^{2}\right)
$$

where $D_{\sigma}\left(p^{2}\right)$ and $D_{\pi}\left(p^{2}\right)$ are the sigma field propagator and pion field respectively, $V_{\pi^{2} \sigma}(p, 0)$ is the three point function with a pion leg at zero external momentum. The second Ward identity can be written as

$$
i F\left[V_{N \pi N}(p, 0)\right]=\frac{1}{2}\left\{\tau_{a} \gamma_{5}, S^{-1}(\not p)\right\} .
$$

In the expression above \{\} means anticomutator, $S(\not p)$ is the nucleon propagator and $V_{N \pi N}(p, 0)$ is another three point function. Only two point functions have quadratic and linear divergences. We therefore conclude that the above two Ward Identities will be enough to verify the one loop cancellation of the integrals containing quadratic and linear divergences.

Let us first analyze the identity in equation (12). The inverse of the pion propagator is given by

$$
D_{\pi}^{-1}\left(p^{2}\right)=p^{2}-\mu^{2}-\Sigma_{R}^{\pi}\left(p^{2}\right)
$$

where

$$
\Sigma_{R}^{\pi}\left(p^{2}\right)=\Sigma_{C T}^{\pi}\left(p^{2}\right)+\Sigma^{\pi}\left(p^{2}\right)
$$

the indices indicate renormalized amplitudes $\mathrm{R}$ and counterterms are indicated by CT. Since the contribution comes from only two diagrams, we write

$$
\Sigma^{\pi}\left(p^{2}\right)=\Sigma_{1}^{\pi}\left(p^{2}\right)+\Sigma_{2}^{\pi}\left(p^{2}\right)
$$

where the indices 1 and 2 indicate the contributions of each diagram. The explicit perturbative expressions for this contributions are given in terms of Feynman amplitudes and can be written as

$$
i \Sigma_{1}^{\pi}\left(p^{2}\right)=8 G^{2}\left[p_{\mu} I_{l i n}^{\mu}\left(p^{2}, m^{2}\right)-I_{\text {quad }}\left(p^{2}, m^{2}\right)\right]
$$

and

$$
i \Sigma_{2}^{\pi}\left(p^{2}\right)=4 F^{2} \lambda^{2} I_{\log }\left(p^{2}, \mu^{2}, M^{2}\right)
$$

where

$$
I_{\text {lin }}^{\mu}\left(p^{2}, m^{2}\right)=\int_{\Lambda} \frac{d^{4} k}{(2 \pi)^{4}} \frac{k^{\mu}}{\left(k^{2}-m^{2}\right)\left[(p-k)^{2}-m^{2}\right]},
$$




$$
I_{\text {quad }}\left(p^{2}, m^{2}\right)=\int_{\Lambda} \frac{d^{4} k}{(2 \pi)^{4}} \frac{1}{\left[(p-k)^{2}-m^{2}\right]}
$$

and

$$
I_{\log }\left(p^{2}, \mu^{2}, M^{2}\right)=\int_{\Lambda} \frac{d^{4} k}{(2 \pi)^{4}} \frac{1}{\left(k^{2}-M^{2}\right)\left[(p-k)^{2}-\mu^{2}\right]}
$$

The index $\Lambda$ in the integral means only an implicit regularization and a specific regulator needs never be used.

We also have the inverse sigma propagator at one loop order.

$$
D_{\sigma}^{-1}\left(p^{2}\right)=p^{2}-M^{2}-\Sigma_{R}^{\sigma}\left(p^{2}\right)
$$

In the same way as before

$$
\Sigma_{R}^{\sigma}\left(p^{2}\right)=\Sigma_{C T}^{\sigma}\left(p^{2}\right)+\Sigma^{\sigma}\left(p^{2}\right)
$$

and since the contribution comes from three diagrams, we write

$$
\Sigma^{\sigma}\left(p^{2}\right)=\Sigma_{1}^{\sigma}\left(p^{2}\right)+\Sigma_{2}^{\sigma}\left(p^{2}\right)+\Sigma_{3}^{\sigma}\left(p^{2}\right) .
$$

Here

$$
\begin{aligned}
i \Sigma_{1}^{\sigma}\left(p^{2}\right) & =8 G^{2}\left[p_{\mu} I_{l i n}^{\mu}\left(p^{2}, m^{2}\right)\right. \\
& \left.-I_{\text {quad }}\left(p^{2}, m^{2}\right)-2 m^{2} I_{\log }\left(p^{2}, m^{2}, m^{2}\right)\right] \\
& i \Sigma_{2}^{\sigma}\left(p^{2}\right)=18 F^{2} \lambda^{2} I_{\log }\left(p^{2}, M^{2}, M^{2}\right)
\end{aligned}
$$

and

$$
i \Sigma_{3}^{\sigma}\left(p^{2}\right)=6 F^{2} \lambda^{2} I_{\log }\left(p^{2}, \mu^{2}, \mu^{2}\right)
$$

The cancellation of the quadratically and linearly divergent contributions can be seen in only the terms $\Sigma_{1}^{\pi}\left(p^{2}\right)$ and $\Sigma_{1}^{\sigma}\left(p^{2}\right)$. Since the integrals $I_{\text {quad }}\left(p^{2}, m^{2}\right)$ and $I_{\text {lin }}^{\mu}\left(p^{2}, m^{2}\right)$ are the same for both contributions $\left(\Sigma_{1}^{\pi}\left(p^{2}\right)\right.$ and $\Sigma_{1}^{\sigma}\left(p^{2}\right)$.) and since the Ward Identity is given by the difference between them, the cancellation is obvious and no specific regularization method is required.

Let us to analyze the second Ward identity (13). In this case we must consider the inverse of the nucleon propagator including its one loop correction

$$
S_{F}^{-1}(\not p)=\not p-m+\Sigma_{R}^{N}(\not p)
$$

(we used the notation $\not p=\gamma^{\mu} p_{\mu}$ ).In an analogous fashion as in the preceding case, we have

$$
\Sigma_{R}^{N}(\not p)=\Sigma_{C T}^{N}(\not p)+\Sigma^{N}(\not p)
$$

and as the contribution comes from two diagrams, we get

$$
\Sigma^{N}(\not p)=\Sigma_{1}^{N}(\not p)+\Sigma_{2}^{N}(\not p)
$$

The explicit perturbative expressions for this contributions are given in terms of Feynman amplitudes

$$
i \Sigma_{1}^{N}(\not p)=G^{2} \int_{\Lambda} \frac{d^{4} k}{(2 \pi)^{4}} \frac{k+m}{\left(k^{2}-m^{2}\right)\left[(p-k)^{2}-M^{2}\right]}
$$

and

$$
i \Sigma_{2}^{N}(\not p)=3 G^{2} \int_{\Lambda} \frac{d^{4} k}{(2 \pi)^{4}} \frac{-\not k+m}{\left(k^{2}-m^{2}\right)\left[(p-k)^{2}-\mu^{2}\right]}
$$

Since $\left\{\gamma^{\mu}, \gamma^{5}\right\}=0$, by the Ward identity (13) one can see the cancellation of the linearly divergent contributions and as before no specific regularization method is required.

\section{THE IMPLICIT REGULARIZATION}

Now a word about the Implicit Regularization Technique which we are using are in order. A simple example of its working procedure can be found in several references [4][5][6], and we give here a simple illustration. In order to illustrate the procedure, consider the following divergent amplitude, typical of one loop order:

$$
\int_{\Lambda} \frac{d^{4} k}{(2 \pi)^{4}} \frac{1}{\left[(k+p)^{2}-m^{2}\right]\left(k^{2}-m^{2}\right)} .
$$

The symbol $\Lambda$ under the integral sign presupposes, as discussed, an implicit regularization. Now, in order to separate the logarithmic divergence from the finite part, we use the following identity in the factor involving the external momentum p:

$$
\begin{aligned}
\frac{1}{\left[(k+p)^{2}-m^{2}\right]} & =\sum_{j=0}^{N} \frac{(-1)^{j}\left(p^{2}+2 p \cdot k\right)^{j}}{\left(k^{2}-m^{2}\right)^{j+1}} \\
& +\frac{(-1)^{N+1}\left(p^{2}+2 p \cdot k\right)^{N+1}}{\left(k^{2}-m^{2}\right)^{N+1}\left[(k+p)^{2}-m^{2}\right]}
\end{aligned}
$$

In the above expression $N$ is chosen so that the last term is finite under integration over $k$. Notice also that in the first term in equation (34), the external momentum appears only in the numerator and thus after integration it can yield at most polynomials in $p$ multiplied by divergences. For our present example we need $N=0$, since we are dealing with a logarithmic divergence. We can rewrite (33) using (34) as

$I=\int_{\Lambda} \frac{d^{4} k}{(2 \pi)^{4}} \frac{1}{\left(k^{2}-m^{2}\right)^{2}}-\int \frac{d^{4} k}{(2 \pi)^{4}} \frac{p^{2}+2 p \cdot k}{\left[(k+p)^{2}-m^{2}\right]\left(k^{2}-m^{2}\right)^{2}}$.

Now only the first of these two integrals is divergent. The others can be easily integrated out to yield

$$
I=I_{l o g}\left(m^{2}\right)-\frac{i}{(4 \pi)^{2}} Z_{0}\left(m^{2}, p^{2}\right)
$$

where

$$
I_{\log }\left(m^{2}\right)=\int_{\Lambda} \frac{d^{4} k}{(2 \pi)^{4}} \frac{1}{\left(k^{2}-m^{2}\right)^{2}}
$$

and

$$
Z_{0}\left(m^{2}, p^{2}\right)=\int_{0}^{1} d z \ln \left(\frac{p^{2} z(1-z)-m^{2}}{-m^{2}}\right) .
$$


Note that, since no explicit form for the regulator has been used, one can make immediate contact with other regularizations. Details of calculations of several one loop amplitudes and their associated Ward identities by using this method can be found in [5].

\section{SCALE CHANGE MECHANISM}

In this section we will present the mechanism of using the relations between mass scales skillfully. We will see how it is possible in this scheme to change scales in the Feynman amplitudes and how this change is intimately connected to the finite parts of these same contributions. In fact, this mechanism is quite general and comes stems from the freedom we have when separating, by means of an identity, a finite from a divergent contribution in a Feynman amplitude. This becomes specially useful and adequate in theories involving different masses, as is the case of the linear sigma model or the SU(3) version of the Nambu and Jona Lasinio model. As we will see, this mechanism is rather essential in order to verify the Ward identities by using the complete amplitude, i.e., divergent plus finite contributions.

Let us begin by using the two definitions already used in the IRT [4]. We start with a definition for basic divergent object. The log divergent quantity :

$$
I_{\log }\left(\xi_{1}^{2}\right)=\int_{\Lambda} \frac{d^{4} k}{(2 \pi)^{4}} \frac{1}{\left(k^{2}-\xi_{1}^{2}\right)^{2}}
$$

as explained in the previous section, the index $\Lambda$ in the integral means only an implicit regularization and $\xi_{1}$ is any mass. Alternatively we can use the identity

$$
\frac{1}{\left(k^{2}-\xi_{1}^{2}\right)}=\frac{1}{\left(k^{2}-\xi_{2}^{2}\right)}-\frac{\left(\xi_{2}^{2}-\xi_{1}^{2}\right)}{\left(k^{2}-\xi_{2}^{2}\right)\left(k^{2}-\xi_{1}^{2}\right)}
$$

and integrate without restrictions the finite integrals in order to obtain the identity

$$
I_{\log }\left(\xi_{1}^{2}\right)=I_{\log }\left(\xi_{2}^{2}\right)-\frac{i}{(4 \pi)^{2}} \ln \left(\frac{\xi_{1}^{2}}{\xi_{2}^{2}}\right) .
$$

Notice that in a theory without mass an infra-red regulator can always be introduced using the identity (40) with $\xi_{1}=0$. The function which identifies all one loop finite parts can always be written as

$Z_{0}\left(\xi_{1}^{2}, \xi_{2}^{2}, p^{2} ; \xi_{2}^{2}\right)=\int_{0}^{1} d x \ln \left(\frac{p^{2} x(1-x)+x\left(\xi_{1}^{2}-\xi_{2}^{2}\right)-\xi_{1}^{2}}{\left(-\xi_{2}^{2}\right)}\right)$

where the function $Z_{0}\left(\xi_{1}^{2}, \xi_{2}^{2}, p^{2} ; \xi_{2}^{2}\right)$ is defined having $\xi_{2}$ as mass scale and then we can use the identity

$$
Z_{0}\left(\xi_{1}^{2}, \xi_{2}^{2}, p^{2} ; \xi_{2}^{2}\right)=Z_{0}\left(\xi_{2}^{2}, \xi_{1}^{2}, p^{2} ; \xi_{1}^{2}\right)+\ln \left(\frac{\xi_{1}^{2}}{\xi_{2}^{2}}\right)
$$

to change the scale of the functions. For an arbitrary scale $\eta$ we will have

$$
Z_{0}\left(\xi_{1}^{2}, \xi_{2}^{2}, p^{2} ; \eta^{2}\right)=Z_{0}\left(\xi_{1}^{2}, \xi_{2}^{2}, p^{2} ; \xi_{2}^{2}\right)+\ln \left(\frac{\xi_{2}^{2}}{\eta^{2}}\right)
$$

The final result of the IRT manipulation for integral

$$
\int_{\Lambda} \frac{d^{4} k}{(2 \pi)^{4}} \frac{1}{\left(k^{2}-\xi_{1}^{2}\right)\left[(p-k)^{2}-\xi_{2}^{2}\right]}
$$

can be written as

$$
I_{\log }\left(\xi_{2}^{2}\right)-\frac{i}{(4 \pi)^{2}} Z_{0}\left(\xi_{1}^{2}, \xi_{2}^{2}, p^{2} ; \xi_{2}^{2}\right)
$$

or

$$
I_{\log }\left(\xi_{1}^{2}\right)-\frac{i}{(4 \pi)^{2}} Z_{0}\left(\xi_{2}^{2}, \xi_{1}^{2}, p^{2} ; \xi_{1}^{2}\right)
$$

or

$$
I_{\log }\left(\eta^{2}\right)-\frac{i}{(4 \pi)^{2}} Z_{0}\left(\xi_{2}^{2}, \xi_{1}^{2}, p^{2} ; \eta^{2}\right)
$$

Let us now proceed to the explicit verification of the Ward identities (12) and (13) and show that an exchange of mass (scale) in the finite part (43) precisely corresponds to a mass exchange in the counterterms (41). After all calculations we can write the one loop Ward identity (12) as

$$
\begin{gathered}
-16 m^{2} G^{2}\left[I_{\log }\left(m^{2}\right)-\frac{i}{(4 \pi)^{2}} Z_{0}\left(m^{2}, m^{2}, p^{2} ; m^{2}\right)\right] \\
+F^{2} \lambda^{2}\left\{18\left[I_{\log }\left(M^{2}\right)-\frac{i}{(4 \pi)^{2}} Z_{0}\left(M^{2}, M^{2}, p^{2} ; M^{2}\right)\right]\right. \\
+6\left[I_{\log }\left(\mu^{2}\right)-\frac{i}{(4 \pi)^{2}} Z_{0}\left(\mu^{2}, \mu^{2}, p^{2} ; \mu^{2}\right)\right] \\
\left.-4\left[I_{\log }\left(M^{2}\right)-\frac{i}{(4 \pi)^{2}} Z_{0}\left(\mu^{2}, M^{2}, p^{2} ; M^{2}\right)\right]\right\}
\end{gathered}
$$

must be equal to

$$
\begin{gathered}
-16 m^{2} G^{2}\left[I_{\log }\left(m^{2}\right)-\frac{i}{(4 \pi)^{2}} Z_{0}\left(m^{2}, m^{2}, p^{2} ; m^{2}\right)\right] \\
+F^{2} \lambda^{2}\left\{\left[14 I_{\log }\left(M^{2}\right)+6 I_{\log }\left(\mu^{2}\right)\right]\right. \\
+\frac{i}{(4 \pi)^{2}}\left[4 Z_{0}\left(\mu^{2}, M^{2}, p^{2} ; M^{2}\right)\right.
\end{gathered}
$$

$$
\left.\left.-18 Z_{0}\left(M^{2}, M^{2}, p^{2} ; M^{2}\right)-6 Z_{0}\left(\mu^{2}, \mu^{2}, p^{2} ; \mu^{2}\right)\right]\right\}
$$

Note that in the last term of eq.(49) we have the freedom to write it as

$$
-4\left[I_{\log }\left(\mu^{2}\right)-\frac{i}{(4 \pi)^{2}} Z_{0}\left(M^{2}, \mu^{2}, p^{2} ; \mu^{2}\right)\right]
$$

One can easily verify that whatever the choice of scale (including an arbitrary one), in the finite part by the relation (43), 
the counterterms are automatically adjusted by the relation (41) in such a way that the Ward identity is always completely satisfied. This becomes more clear in the Ward Identity (13), which can be expressed as

$$
\begin{gathered}
\tau_{a} \gamma_{5} m G^{2}\left\{3\left[I_{\log }\left(m^{2}\right)-\frac{i}{(4 \pi)^{2}} Z_{0}\left(\mu^{2}, m^{2}, p^{2} ; m^{2}\right)\right]\right. \\
\left.-\left[I_{\log }\left(m^{2}\right)-\frac{i}{(4 \pi)^{2}} Z_{0}\left(M^{2}, m^{2}, p^{2} ; m^{2}\right)\right]\right\}
\end{gathered}
$$

which must be equal to

$$
\begin{gathered}
\tau_{a} \gamma_{5} m G^{2}\left\{\left[I_{\log }\left(m^{2}\right)-\frac{i}{(4 \pi)^{2}} Z_{0}\left(M^{2}, m^{2}, p^{2} ; m^{2}\right)\right]\right. \\
+\left[I_{\log }\left(m^{2}\right)-\frac{i}{(4 \pi)^{2}} Z_{0}\left(\mu^{2}, m^{2}, p^{2} ; m^{2}\right)\right] \\
\left.+\frac{i}{(4 \pi)^{2}} 2\left[Z_{0}\left(M^{2}, m^{2}, p^{2} ; m^{2}\right)-Z_{0}\left(\mu^{2}, m^{2}, p^{2} ; m^{2}\right)\right]\right\}
\end{gathered}
$$

where we have used the fermion mass for commodity only.

\section{CONCLUSION}

In the calculation it becomes apparent the delicate and essential connection between divergent and finite parts of amplitudes and the examples show how to use mass scales identities which are absolutely necessary to manipulate graphs involving several masses in a way as to show its equivalence to others involving different (than the previous) masses.

One of the advantages of the present technique is that we have all counterterms in an explicit form. This simplifies the renormalization procedure, since this can be done directly in the Lagrangian. The renormalization procedure due to some symmetry require counterterms with the same mass and then we can introduce the arbitrary scale, which has been shown to play the role of the sliding scale of the theory (see Ref.[5]).

\section{Acknowledgments}

The work of M.C. Nemes was partially supported by CNPq. S.R. Gobira would like to thank C. M. G.de Sousa and Paulo H. L. Martins for some discussions.
[1] Guy Bonneau, J. Mod. Phys. A 520, 3831 (1990)

[2] G. 't Hooft and M. Veltman, Nucl. Phys. B 44, 189 (1972). G.'t Hooft and M. Veltman, Nucl. Phys. B 50, 318 (1972). C.G. Bollini and J.J. Giambiagi, Nuovo Cimento B 12, 20 (1972).

[3] D. Z. Freedman, K. Johnson, and J. I. Latorre, Nucl. Phys B 371, 353 (1992). F. del Águila, A. Culatti, R. Muñoz Tápia, and M. Pérez -Victoria, Nucl. Phys. B 537, 561 (1999); Phys. Lett. B 419, 263 (1998); Nucl. Phys. B 504, 532 (1997). M. Pérez -Victoria, Nucl. Phys. B 442, 315 (1998).

[4] O. A. Battistel, - UFMG - PhD Thesis (1999). O. Battistel, M. C. Nemes, Symmetry Relations in The N.J.L. Model. Phys. Rev. D 59, 055010 (1999). O. A. Battistel, A. L. Mota, and M. C. Nemes, Mod. Phys. Lett. A 13, 1597 (1998).

[5] M. Sampaio, A. Scarpelli, B. Hiller, A. Brizola, M. C. Nemes, and S. R. Gobira, hep-th/0203261. Phys. Rev. D 65, 125023
(2002). A. Brizola, S. R. Gobira, M. Sampaio, and M. C. Nemes, Int. Jour. Theor. Phys. 41, 9 (2002). A. P. Scarpelli, M. Sampaio, and M.C. Nemes, Phys Rev. D 63, 046004 (2001).

[6] N-loop Treatment of Overlapping Diagrams by the Implicit Regularization Technique, S. R. Gobira and M. C. Nemes, hepth/0102096, Int. Jour. Theor. Phys. 42, 11 (2003).

[7] S. R. Gobira, O. A. Battistel, and M. C. Nemes, Braz. Journ. Phys.30-3, 610 (2000).

[8] D.Bessis and G. Turchetti: Cargèse Lectures in Physics 5 (Gordon and Breach. New York, 1971) 119.

[9] J. Schwinger, Ann. Phys. 2, 407 (1957).

[10] M. Gell-Mann and M. Levy, Nuovo Cimento 16, 705 (1960).

[11] J.C. Polkinghorne, Nuovo Cimento 8, 179, 781 (1958). 